\title{
Comparison of different knee states: intact, without A.C.L. and with a prosthetic using a kinematics simulator
}

\author{
A. Bouafia ${ }^{1} \&$ J. Dimnet ${ }^{2}$ \\ ${ }^{1} C M P / F G M G P /$ University of Sciences and Technologies Houari \\ Boumedienne, Alger \\ ${ }^{2}$ LBM/CM/ University of Claude Bernard Lyon, France
}

\begin{abstract}
The purpose of this paper is to compare the kinematics of the same knee joint in different successive states: intact, after A.C.L. (Anterior Cruciate Ligament) ruptures, and then replaced by a prosthetic implant. To attain this objective required working on a cadaver limb, using a speciality designed simulator to reproduce continuous flexion-extension cycles under realistic loading conditions.

The successive positions of the knee adjacent segments (femur and tibia) are measured using a motion analysis system that computes the three dimensional trajectories of a set of reflective markers screwed directly into the bones. The motion analysis system is made up from the following components: a SUN workstation, five video cameras, an electronic interface that allows the conversion of the optical signal into an electronic signal and the synchro between the different signals (force, electro-myograph ....), and one software set for 3D reconstruction. We then apply the theory of solid kinematics in order to calculate the relative displacement between the two adjacent segments, assumed to correspond to the knee joint movement. Specific tests have been executed in order to quantify the errors due to the measurement protocol, so that the results obtained for the various knee states under scrutiny can be assigned to the actual changes in the functioning joint.

In this paper we first describe the experimental material and conditions, then the method used to assess the joint kinematics and then quantifying the errors due to the measurement protocol is explained. Finally, the most representative results of the comparison between the different knee states are displayed and commented on from a clinical point of view.
\end{abstract}

Keywords: anatomic piece, optoelectronic system, simulator, knee kinematics, three dimensional motion, the finite helical axis. 


\section{Introduction}

The literature relative to the knee's behaviour is notoriously sizeable, and the means of investigation are numerous; two or three dimensional radiography, scanner, I.R.M., video-based systems [1], are a few among the many tools available to the researcher.

The optoelectronic systems SELSPOT [2, 3], ELITE [4], VICON [5], MOTION ANALYSIS [6] allow us to study the joint's displacements in the 3D space. During the last few years their usage has increased steadily, for in vivo as well as for in vitro research. Their principle is to give the spatial trajectories of markers fixed on the subject's body segments during a given movement. In order to use the theory of solid kinematics, the body segments are assumed to be rigid and the relative displacement between two adjacent segments are then construed (supposed) to represent the joint's movement.

For in vivo experiments, the markers are often glued directly on the skin, and the above mentioned hypothesis becomes incorrect because of the perturbations on the marker displacements due to the skin elasticity, the interposed muscle and the fat between the fixed markers on the skin and the underlying bones. These perturbations introduce important errors on the measured positions of the adjacent segments, and even more so on joint's kinematics calculations.

Under these experimental conditions, the marker trajectories have to be corrected first, using either a least-square method [7] or a solidification procedure $[8,9]$.

The aim of the paper is to test pathological effects (Anterior Cruciate Ligament severance or prosthetic implantation) on the knee kinematics. Therefore, we choose to work on cadaver limbs since the previously described perturbations can be avoided by directly screwing the markers on the two adjacent bones in this fashion the measured displacement of the femur relative to the tibia represents correctly the knee joint movement. In order to compare the different knee states under the same conditions, a simulator has been designed to reproduce flexion-extension cycles under loading conditions.

Two kinds of simulator are used in biomechanics. The first one simulates the functioning joint in order to report the abrasion phenomenon: [4, 10]. The other simulates specific tasks of the joint to better understand the joint mechanism: $[10,11]$. The simulator used for our report is of this latter type.

The knee joint flexion-extension movement has been studied over the past years. Some authors produce the knee displacement discontinuously, with a predefined angular magnitude for each movement step $\left(15^{\circ}\right)$ and a good approximation of the real loading conditions $(300 \mathrm{~N}$ are applied on the femoral head: $[12,13]$. Others realise a continuous flexion-extension movement using a motorised system, like Reuben et al [10], but do not take into account realistic in vivo loads (only 40 to $60 \mathrm{~N}$ are applied on the femoral head).

This paper first describes the experimental equipment and conditions, and then the method used to obtain the knee joint kinematics [12]. The most representative results of the comparison between the intact knee, the knee after A.C.L. severance and then prosthetic implantation are finally displayed and commented. 


\section{Equipment and methods}

\subsection{Equipment and experimental protocol}

The knee flexion-extension movement is obtained using a specific simulator, Figure 1. It is composed of a platform, able to translate vertically with respect to the fixed structure, which stroke is controlled by positions transducers mounted on the apparatus, which regulate the stroke (these sensors are convertible in flexion amplitude). This platform supports the motor operating on a cable representing the quadriceps muscle action braking the descent and controlling the upward motion. The anatomic specimen is a lower limb taken from a fresh cadaver presenting no pathology. The skin and muscles are removed to screw directly the markers on bones, except around the knee joint where the whole ligamentary structure remains intact in order to conserve their role during the simulation period. The femur is jointed with the platform by a hip prosthesis ( 3 rotational degree of freedom) and the base of the tibia is cut at the tibio-tarsienne articulation, a metal piece is put on the middle zone (central) of the bone, the other extremity is assembled on an articulated pivot, ( 01 rotational degree of freedom) this mounting leaves 3 rotational axis for the internal structure of the anatomic knee.

The charge of $400 \mathrm{~N}$ is supposed centred on the plate form and applied to the head of the femur (hip joint) to simulate the loading conditions of the flexion movement effected by a living subject.

In order to follow the movement using a motion analysis system, four reflective markers are screwed directly in each bone, Figure 2 . Their successive positions are collected during the simulated movement by five cameras whose sampling frequency is set to $60 \mathrm{~Hz}$. Then, software based on D.L.T. (Direct Linear Transform) is used to compute the $3 \mathrm{D}$ trajectories of the markers. Three data collections are completed with the intact knee, each one corresponding roughly to three movement cycles. Then, the A.C.L. is cut without dismantling of the system, and three other set of data are executed. Finally, the limb is taken off and the markers are removed in order that the surgeon can set the prosthetic implant. The knee joint is replaced by a H.L.S.II prosthesis (constructed by Tornier S.A.). HLSII is a prosthesis that includes a central stop with a translation limiting function, the limit in the direction of the antero-posterior axis during the flexion of the knee.

The stability of the femur forward movement is achieved in this kind of prosthesis by the tibial plate concavity. This concavity limits the knee axial rotation and translation starting from $35^{\circ}$ of flexion, and a stabilising cam also locks the forward translation of the femur with respect to the tibia, assuming the A.C.L. play as the cruciate ligaments are taken off for this kind of implant. When the knee prosthesis is set up, the limb is mounted in the simulator, the markers are screwed again in the same locations and three last data are executed. The locations of the markers, displayed on Pict.3, are chosen to build a reference frame affixed to each bone, which axes correspond to anatomic directions. 


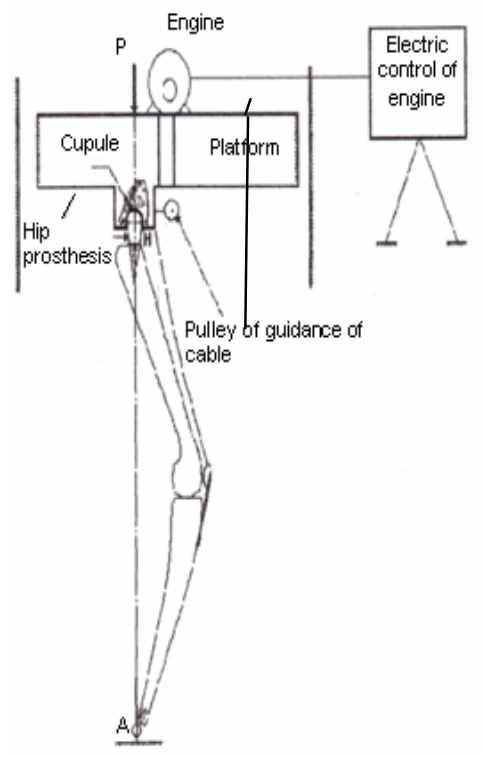

Figure 1: Simplified schema of simulator.

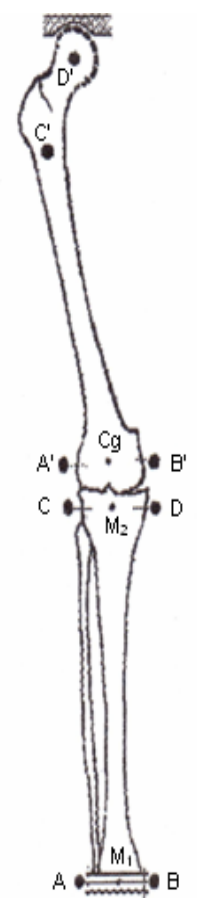

Figure 2: Disposition of markers on tibia and femur.

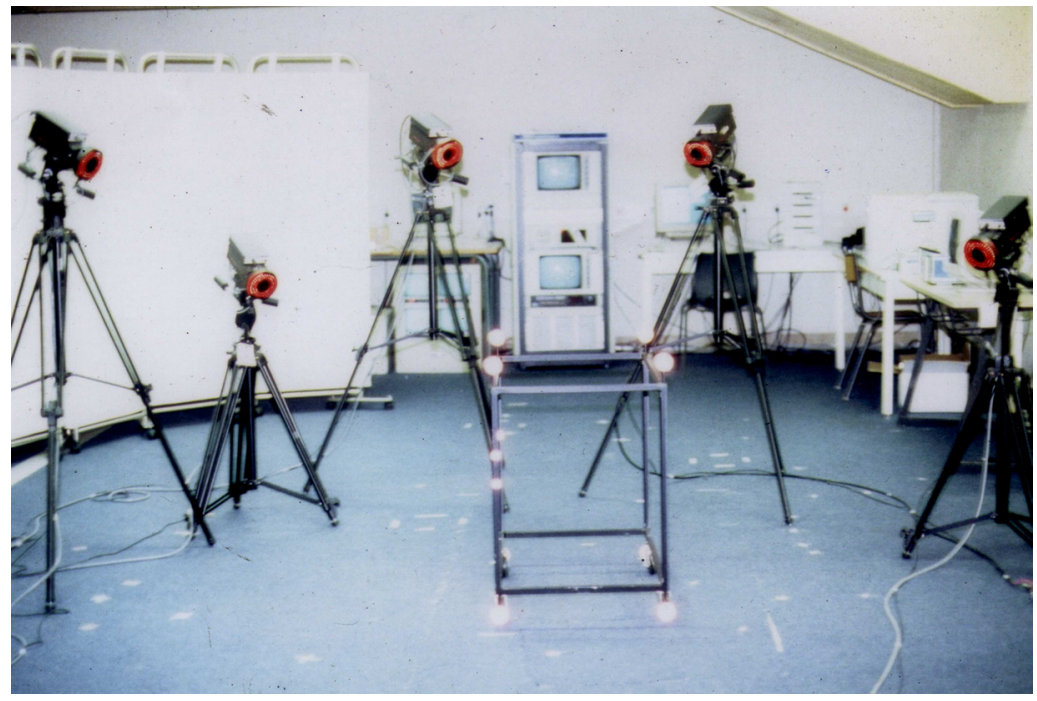

Figure 3: An optoelectronic system. 


\subsection{Method}

For the tibia, the $X_{t}$ axis joints markers $\mathrm{C}$ and $\mathrm{D}$. The $Z_{t}$ axis is perpendicular to the plane formed by $X_{t}$ and the longitudinal direction materialised by M1M2: M1 and M2 being respectively the midpoints of $[A, B]$ and $[C, D]$. The $Y_{t}$ axis is finally obtained by calculating the cross-product: $Y_{t}=Z_{t} \times X_{t}$ so that $\left(X_{t}, Y_{t}, Z_{t}\right)$ constitutes a direct trihedron. The reference frame $\left(X_{f}, Y_{f}, Z_{f}\right)$ affixed to the femur is defined similarly [14].

From the successive positions of these two reference frames, it is possible to determine the relative displacement of the tibia with respect to position which corresponds to the fully extended knee, named 1) and a current position, named i [8].

Let ${ }_{t i}^{0} R$ and ${ }_{t i i}^{0} R$ be the rotation matrices defining the orientation of the tibia frame respectively in the initial and current position, with respect to the fixed laboratory frame Ro.

Let ${ }_{f 1}^{0} R$ and ${ }_{f i}^{0} R$ be the rotation matrices defining the orientations of the femur frame at the same instants. The matrix ${ }_{i t}^{t 1} R$ describing the relative rotation of the tibia with respect to the femur assumed fixed femur is obtained by superimposing the femur frames in position 1 and i. So, the matrix ${ }_{t i}^{t 1} R$ is computed, for each successive position $\mathrm{i}$, using the following relation:

$$
{ }_{t i}^{t 1} R=\left[{ }_{t 11}^{00} R\right] \cdot\left[{ }_{f 1}^{0} R\right] \cdot\left[{ }_{f i}^{0} R\right]^{-1} \cdot\left[{ }_{t i}^{0} R\right]
$$

The rotation of the tibia relative to the femur is composed in three successive rotations about fixed axis. The first rotation, around $X_{t 1}$, moves the tibial frame in a given orientation (intermediary frame $R_{t}$ ). The following rotation, around $Z_{t 1}$, moves $\mathrm{R}_{\mathrm{t}}$, in a new position $\mathrm{R}_{\mathrm{t}}$ ". The last rotation around $Y_{t 1}$ moves the previous frame $\mathrm{R}_{\mathrm{t}}$, in the final tibial orientation $\mathrm{R}_{\mathrm{ti}}$. The analytic form of the operator corresponding to these three successive rotations about fixed axes (coinciding to those of the tibial reference frame in the initial position) is obtained as follows:

$$
\begin{gathered}
R_{t 1} \stackrel{\operatorname{rot}\left(X_{t 1}, \theta_{1}\right)}{\longrightarrow} R_{t^{\prime}} \stackrel{\operatorname{rot}\left(Z_{t 1}, \theta_{2}\right)}{\longrightarrow} R_{t^{\prime \prime}} \stackrel{\operatorname{rot}\left(Y_{t 1}, \theta_{3}\right)}{\longrightarrow} R_{t i} \\
{ }_{t i}^{t 1} R=\left[\begin{array}{ccc}
\cos \theta_{3} & 0 & \sin \theta_{3} \\
0 & 1 & 0 \\
-\sin \theta_{3} & 0 & \cos \theta_{3}
\end{array}\right] \cdot\left[\begin{array}{ccc}
\cos \theta_{2} & -\sin \theta_{2} & 0 \\
\sin \theta_{2} & \cos \theta_{2} & 0 \\
0 & 0 & 1
\end{array}\right] \cdot\left[\begin{array}{ccc}
1 & 0 & 0 \\
0 & \cos \theta_{1} & -\sin \theta_{1} \\
0 & \sin \theta_{1} & \cos \theta 1
\end{array}\right]
\end{gathered}
$$




$$
\left({ }_{t i}^{t 1} R\right)=\left(\begin{array}{ccc}
c 2 c 3 & s 3 s 1-c 1 s 2 c 3 & s 1 s 2 c 3+s 3 c 1 \\
s 2 & c 1 c 2 & -s 1 c 2 \\
-c 2 s 3 & c 1 s 2 s 3+c 3 s 1 & c 3 c 1-s 1 s 2 s 3
\end{array}\right)=\alpha i j
$$

From the identification between the numerical values of the ${ }_{t i}^{t 1} R$ coefficients $\alpha_{i j}$, computed from the 3D trajectories of the markers (equation 1), and the analytic form of the same coefficients, the following relations can be deduced:

$$
\left\{\begin{array}{c}
\theta_{1}=\tan ^{-1}\left(-\alpha_{23} / \alpha_{22}\right) \\
\theta_{2}=\tan ^{-1}\left(\alpha_{21} / \sqrt{\alpha^{2}{ }_{22}+\alpha^{2}{ }_{23}}\right) \\
\theta_{3}=\tan ^{-1}\left(-\alpha_{31} / \alpha_{11}\right)
\end{array}\right.
$$

These angles correspond to successive elementary rotations about the three axes of the tibial reference frame in the initial position (full extended). This combination has been chosen because the angular values so obtained are easily transposed by clinicians as a flexion-extension component $\theta_{1}$, followed by an abduction-adduction $\theta_{2}$ and finally an axial rotation $\theta_{3}$, moving the knee joint from its initial position 1 to its current position i. These angles are of high interest for the analyses of prostheses. The component around $X_{t 1}$ and $Z_{t 1}$ correspond to rolling (respectively for flexion and abduction movements) and the components around $Y_{t 1}$ represents the pivoting (axial rotation $\theta_{3}$ ). A direct relation exists between the abrasion of the prosthesis contact surfaces and the pivoting component, so the movement's description proposed is well adapted to define to working conditions of a prosthetic implant. This approach is interesting for the analysis of the behaviour of the prosthesis instead of the subjects. There is a direct relation between the wear of the prosthesis and the pivoting component (that corresponds to the amplitude of the axial rotation). Experience showed that the resistant couple while pivoting is directly opposed to the instantaneous value of the pivoting angular variation [15].

Because the knee translation is an important clinical parameter in static conditions, it appeared interesting to measure the evolution of this translation during the simulated movement. The knee translation is defined by the displacement of the projection of the geometrical knee center (midpoint of markers A',B' fixed on the knee epicondyles) onto the tibial plate, between the initial position 1 and the current position i. [12].

\section{Results}

Four tests were completed on atomic pieces with different types of knee: intact knee, knee without LCA and instrument layed knee with prosthesis, we represent the results in a lumped case, which represent all the cases tested: 
1. The prosthesis stabilised-posterior HLS II restores approximately the movement extension-flexion of the safe knee. The regularity of the cycle is more remarkable for the prosthesis knee. The good quality of the design of the prosthesis and the conditions of installation may be the cause of this performance. The extension-flexion cycle inversion time is longer in the case of safe knee than is the case of the prosthesis knee. This is due to the fact that the prosthesis has no or a little damping if any for the safe knee the articulate surfaces and ligaments crossed play a role on the damping of the movement especially on the end of the cycle (graph C1).

2. For the knee without LCA, the axial rotation is weaker, it's about $5^{\circ}$ and the cyclic phenomenon is less remarkable (graph C2). It's important to realise that when the LCA is cut, the amplitude of this rotation is important at rest. In movement, the amplitude of this rotation is relatively small, this may be explained by the gliding movement of the femur with respect to the tibia, and the increase of the pressure efforts made on the articulation surfaces during the flexion. In our opinion, these 2 factors limit the axial rotation under dynamic condition.

3. In the case of the tibial translation on the sagital plan, the maximum amplitude is observed for a knee without LCA, the observed translations peaks correspond to the instable rapid gliding movement of the femur with respect to the tibia they are remarkable starting from certain angle of flexion. For the prosthesis knee, the amplitude of translation is nearly nil. The fluctuations observed on the graph (C4) are due to the uncertainty of measure system.

\section{Conclusions}

This paper presents a comparison between the kinematics of three different states of the knee joint: intact, after A.C.L. severance and when replaced by a prosthetic implant. In order to complete this research we have had to work on a cadaver limb, and we had to specifically design a simulator capable of emulating in vivo conditions. The following factors are worth noticing, the flexionextension movement was continuous, a weight of $400 \mathrm{~N}$ was applied on the femoral head, and the quadriceps muscle action was taken into account. Although the experimental conditions included in vivo movement (only one D O $\mathrm{F}$ allocated to the ankle joint, only the quadriceps muscle were taken into account), the protocol was defined as rigorously as possible so that the experiments can be considered reproducible enough to allow a qualitative comparison. This report is undoubtedly an essential complement to this research deals with a first attempt to understand the influence of specific knee pathologies. Attractive results are being obtained; it seems interesting to go further in this research by simulating other kinds of pathologies. Finally, it should be necessary to improve the simulator mechanism, in order to better assess the in vivo conditions: evolution of the ankle joint with additional possibilities of rotation of small magnitude, materialisation of other muscle actions (jumeaux, ischio-jambiers) in order to represent their stabilising effect. 


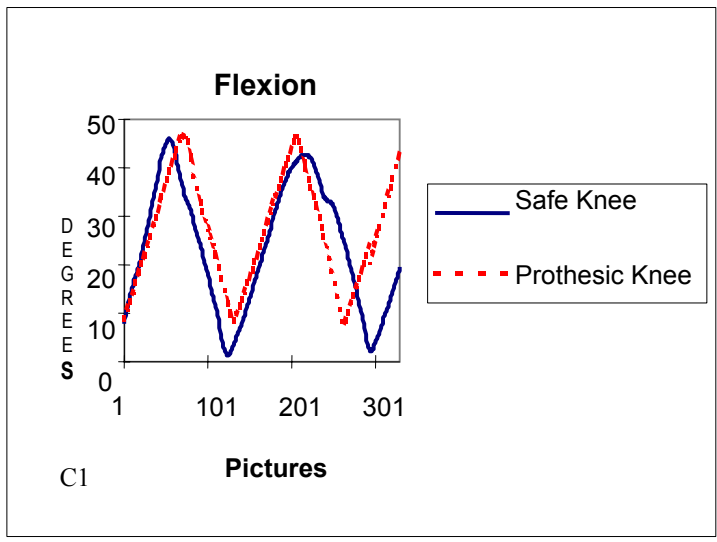

Figure 4: Flexion.

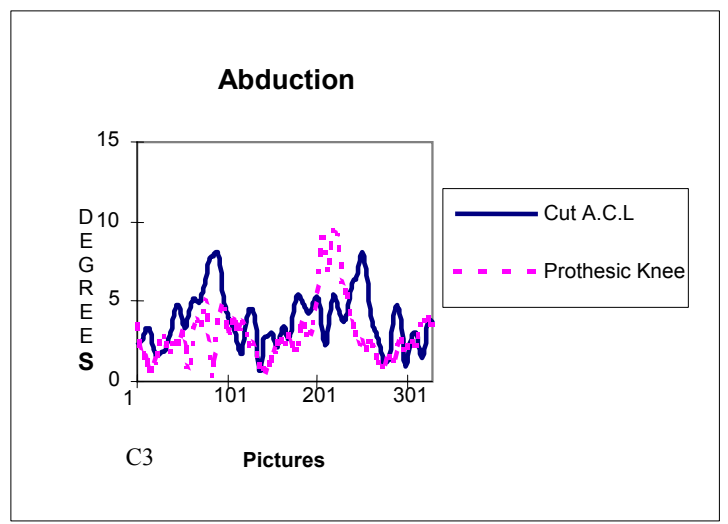

Figure 5: Abduction.

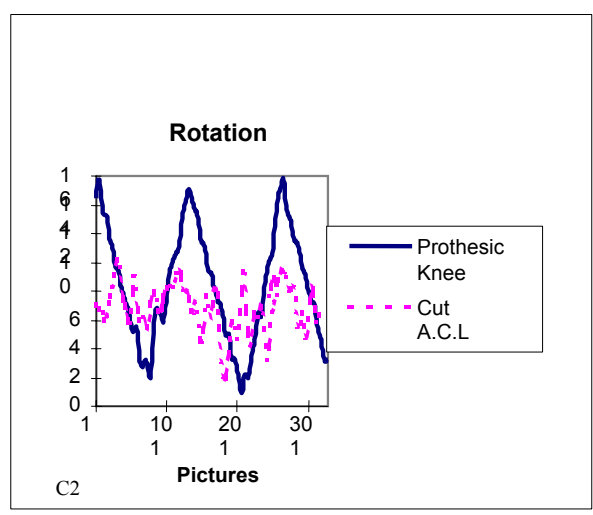

Figure 6: Rotation. 


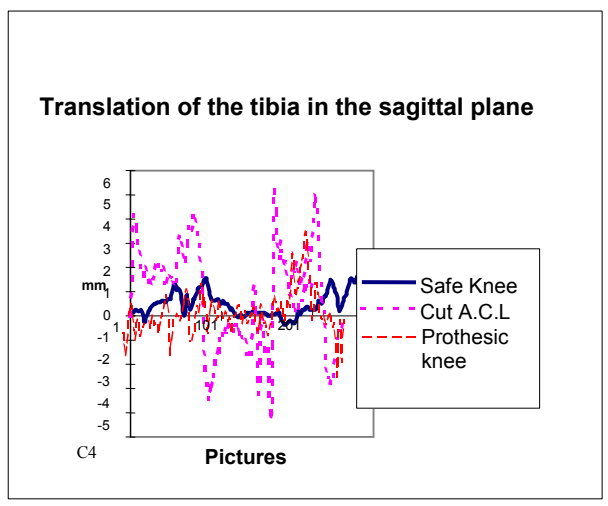

Figure 7: Translation of the tibia in the sagittal plane.

\section{References}

[1] R.K. Begg., R. Wytch, R.E. Major, Instrumentation used in clinical gait analysis:

[2] T.P. Andriacchi, Clinical applications of the SELSPOT system. Biomechanics Symposium ASME, vol. 84, p.339-342., 1987.

[3] T.P. Andriacchi, O. Galante and W. Joig, Fermier Rex., The influence of total knee replacement of the lower limb. J. Biomechanics $22 \mathrm{n}^{\circ} 2, \mathrm{p} 143$ $155,1982$.

[4] A. Pedotti, P. Crenna, A. Deat, C. Frigo, Massion, Postural synergies in axial movements: short and long term adaptation. Exp. Brain Res., vol.74, p.3-10,1989.

[5] F. Pelisse., F. Marie, Conception des prothèses articulaires, biomécanique et biomatériaux. Cahiers d'enseignement de la SOFCOT, n44, p.135-140, 1993.

[6] P. Allard, J.A.F. Stokes, J.P. Blanchi, Three dimensional analysis of human movement. Human kinetics 371 p, 1994.

[7] I. Söderkvist, P. Wedin, Determining the movements of the skeleton using well-configured markers. J. Biomechanics, vol. 26, p. 1473-1477,. 1993.

[8] L. Chèze, B.J. Fregly, J. Dimnet, A solidification procedure to facilitate kinematic analyses based on video system data. J. Biomechanics, vol. 28, $\mathrm{n}^{\circ} 7$, p. 879-884, 1995.

[9] L. Chèze, B.J. Fregly, J Dimnet, Determination of joint functional axes from noisy markers data using the finite helical axis. Human Movement Science 17, 1-15; 1998.

[10] J.D. Reuben, J.S. Rovick, R.J. Schrager, P.S. Walker, A.L. Bollang, Three dimensionnal dynamic motion analysis of the anterior cruciate ligament deficient knee joint. Am. J. Sport med., vol. 17, n²4, p. 463-471, 1989. 
[11] B. Landjerit, M. Bisserie, Cinématique spatiale de l'articulation fémorotibiale du genou humain, caractérisation expérimentale et implication chirurgicales. Acta orthopaedica Belgica, vol. 58, p. 147-158, 1992.

[12] M. Bonnin, La subluxation tibiale anterieure en appui monopodal dans les ruptures du L.C.A., étude clinique et biomécanique. Thèse médecine, Université Lyon I (France), 1990.

[13] V. Djordjalian, Contribution à l'étude biomécanique des articulations humaines, modélisations tridimentionnelles de la colonne vértébrale et du genou. Thèse de Doctorat Université Lyon I, France, 1991.

[14] A. Bouafia, Cinematique du genou; approches in-vivo et in-vitro Revue du CDTA "Technologies Avancées N 13 juillet 2001.

[15] P. Agati, M. Rosseto, Liaisons et mecanismes. Ed. Dunod, 1994. "a review. J. of Medical Engineering and Technology, vol. 13, n 6 p. 290295,1989 